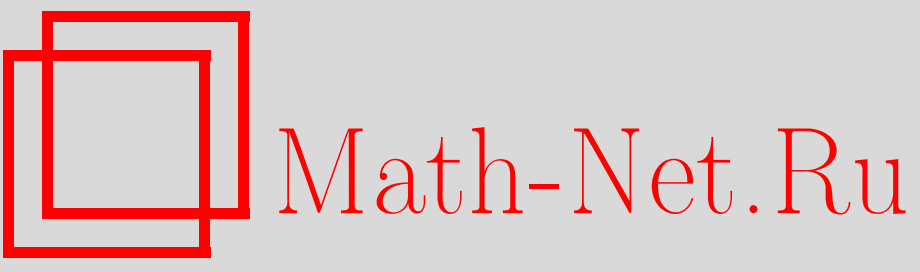

Е. В. Щепин, Об отображениях двумерной сферы, УМН, 2003, том 58, выпуск $6,169-170$

DOI: https://doi.org/10.4213/rm691

Использование Общероссийского математического портала Math-Net.Ru подразумевает, что вы прочитали и согласны с пользовательским соглашением

http://www.mathnet.ru/rus/agreement

Параметры загрузки:

IP : 54.224 .60 .19

26 апреля 2023 г., $07: 13: 16$ 


\title{
ОБ ОТОБРАЖЕНИЯХ ДВУМЕРНОЙ СФЕРЫ
}

\author{
Е. В. ШЕпин
}

Х. Хопф в работе [1] доказал, что для всякого отображения $f: X \rightarrow X$ локально связного уникогерентного континуума $X$ и для всякого отображения $g: X \rightarrow Y$ в одномерньй континуум $Y$ найдется $x \in X$, для которой $g(x)=g(f(x))$. Если в этой теореме в качестве $X$ взять двумерную сферу $S^{2}$, а в качестве $f$ - инволюцию, то теорема Хопфа влечет, что всякое отображение из $S^{2}$ в одномерный континуум склеивает пару антиподов. Этот же результат нетрудно получить из теоремы Шклярского [2] о том, что для любого конечного, кратности 2, замкнутого покрытия сфферы $S^{2}$ и любого отображения $f: S^{2} \rightarrow S^{2}$ найдется $x \in S^{2}$, для которой $x$ и $f(x)$ принадлежат одному и тому же элементу покрытия. В настоящей заметке эта теорема Хопфа-Шклярского обобщается на отображения в компакты нижней размерности единица.

Нижней размерностью компакта назьваем минимум из его размерностей по всем группам коэффициентов. Накрытием называем локально-тривиальное расслоение с дискретньм слоем. Континуум $X$ назьваем ненакрыв ваемым, если он не имеет нетривиалшных (отличных от гомеоморфиимов) связных накрытий. Другими словами, всякое накрытие ненакрываемого компакта допускает сечение.

Лемма 1. Пусть $f: X \rightarrow Y$ - непрерывное сюрбективное отображение ненакрываемого компакта $X$.И пусть $p: Y^{\prime} \rightarrow Y$ - накрытие. Тогда существует непрерьвное отображение $f^{\prime}: X \rightarrow Y^{\prime}$ такое, что $f=p f^{\prime}$. Если $p$ не имеет сечений, то ограничение $p$ на образ $f^{\prime}(X)$ не инвективно.

ДокаЗАТЕЛЬСтво. Пусть $Z=\left\{(x, y) \in X \times Y^{\prime} \mid f(x)=p(y)\right\}$. Тогда ограничение проекции произведения $X \times Y^{\prime} \rightarrow X$ на $Z$ является локальным гомеоморфизмом и в силу ненакрываемости $X$ допускает сечение. Композиция этого сечения с проекцией произведения $X \times Y^{\prime} \rightarrow Y^{\prime}$ и является искомым отображением $f^{\prime}$. Если же ограничение $p$ на образ инъективно, то формула $y \rightarrow f^{\prime}\left(f^{-1}\right)(y)$ задает однозначное непрерывное отображение, являющееся сечением для $p$.

Если для любой группы $G$ выполняется неравенство $\operatorname{dim}_{G} X \leqslant \operatorname{dim}_{G} Y$, то говорят, что $X$ имеет меньший, чем $Y$, размерностный тип, и пишут $\operatorname{DIM} X \leqslant \operatorname{DIM} Y$ (см. [3]).

Лемма 2. Для любого непрерывного отображения $f: X \rightarrow Y$ ненакрываемого компакта $X$ существуют ненакрываемый компакт $Y^{\prime}$ размерностного типа DIM $Y^{\prime} \leqslant$ $\operatorname{DIM} Y$ и такие отображения $f^{\prime}: X \rightarrow Y^{\prime}$ u $c: Y^{\prime} \rightarrow Y$, что $f=c f^{\prime}$.

ДокАЗАТЕЛЬСтво. По трансфинитной индукции будем строить обратньй спектр компактов $\left\{Y_{\alpha}, c_{\alpha, \beta}\right\}$, в котором короткие проекции $c_{\alpha+1, \alpha}$ являются погружениями (локально инъективны), и трансфинитную последовательность отображений $f_{\alpha}: X \rightarrow Y_{\alpha}$ так, что $c_{\alpha, \beta} f_{\alpha}=f_{\beta}$ при любых $\alpha>\beta$. Заметим, что в этом случае $\operatorname{dim}_{G} Y_{\alpha} \leqslant \operatorname{dim}_{G} Y$ при любом $\alpha$, поскольку погружения не уменьшают, а предельные переходы не повышают размерностей.

Полагаем $Y_{1}=Y$ и $f_{1}=f$. Пусть компакты и отображения с индексами $\leqslant \alpha$ уже построены. Если компакт $Y_{\alpha}$ не имеет накрытий, то полагаем $Y^{\prime}=Y_{\alpha}, f^{\prime}=f_{\alpha}$ и $c=c_{\alpha, 1}$ и лемма доказана. Если же имеется нетривиальное накрытие $p: X^{+} \rightarrow X_{\alpha}$, то в качестве $f_{\alpha+1}$ берем какое-нибудь поднятие $f_{\alpha}$, существующее благодаря лемме 1 , а в качестве $Y_{\alpha+1}$ берем $f_{\alpha+1}(X)$. Проекция $c_{\alpha+1, \alpha}$ определяется как ограничение накрытия $p$. Заметим, что в данном случае отображение $c_{\alpha+1, \alpha}$ не является инъективным, иначе накрытие $p$ имело бы непрерьвное сечение (лемма 1 ).

В случае предельного трансфинита $\alpha$ пространство $Y_{\alpha}$ и отображение $f_{\alpha}$ определяются по непрерывности, т.е. $Y_{\alpha}=\operatorname{invlim}_{\gamma<\beta<\alpha}\left\{Y_{\beta} ; c_{\beta, \gamma}\right\}$ и $f_{\alpha}=\operatorname{invlim}_{\beta<\alpha} f_{\beta}$ и $c_{\alpha, \beta}=\operatorname{invlim}_{\gamma}<\alpha c_{\gamma, \beta}$.

Проведя конструкцию для всех счетных трансфинитов, рассмотрим предел $Y_{\omega}$ получившегося обратного спектра. Этот предел является непрерывньп образом метризуемого компакта $X$ и потому сам является метризуемым компактом. Напомним, что в силу Спектральной теоремы [4] лю-

Работа выполнена при поддержке Российского фонда фундаментальных исследований (грант № 02-01-00014). 
бые два трансфинитных спектра из компактов с гомеоморфными пределами содержат изоморфные подспектры. Применяя это утверждение к паре, состоящей из стационарного спектра, все пространства которого гомеоморфны $X_{\omega}$, и построенного выше, заключаем, что в построенном спектре имеются гомеоморфизмы, чего не может быть. Следовательно, наше построение должно оборваться на каком-то счетном транфините.

ЛЕмма 3. Ненакрываемый пеановский континуум нижней размерности 1 не содержит окружности.

ДокАЗАТЕЛЬСтво. Пусть $X$ имеет размерность 1 по отношению к группе $G$ и содержит компакт $S$, гомеоморфиьй окружности. Пространство Эйленберга-Маклейна $K(G, 1)$ содержит нестягиваемую окружность. Рассмотрим гомеоморфное отображение из $S$ на эту нестягиваемую окружность и продолжим это отображение до отображения $f: X \rightarrow K(G, 1)$. Мы получим отображение из $X$ в $K(G, 1)$, нетривиальное на фундаментальной группе. Рассмотрим pull-back от универсалшного накрытия комплекса Эйленберга-Маклейна относителшно $f$. Полученное накрытие не может быть тривиально. Действительно, в противном случае оно имело бы сечение и наше отображение пропускалось бы через универсальное накрытие и было бы тривиально на фундаменталшной группе.

Лемма 4. Локально связный континуум, не содержащий окружности, одномерен, вложим в плоскость и стягиваем.

ДоКАЗАТЕЛЬСТво. Действительно, ведь континуум с описанными свойствами является дендритом (см. [5]).

Теорема 1. Всякое непрерывное отображение сферы $S^{2}$ в компакт нижней размерности 1 склеивает пару антиподов.

ДокАЗАтЕльство. Так как сфера ненакрьваема, то, благодаря лемме 2, любое отображение $f: S^{2} \rightarrow X$ в компакт нижней размерности 1 представляется в виде композиции $f=g h$, где $h: S^{2} \rightarrow X^{\prime}$ и $X^{\prime}$ ненакрьваем. Так как $X^{\prime}$ является пеановским как образ сферы, то в силу леммы $3 X^{\prime}$ - дендрит. А так как всякий дендрит - плоский (лемма 4 ), то $h$, а значит и $f$, склеивает пару антиподов по теореме Борсука-Улама.

Так как всякий дендрит стягиваем, то приведенное вьше доказательство позволяет получить дополнительно такой резултат.

ТЕОРема 2. Континуум нижней размерности 1 асферичен.

\section{СПИСОК ЛИТЕРАТУРЫ}

[1] H. Hopf // Fund. Math. 1937. V. 28. Р. 33-57. [2] Д. О. Шклярский // Матем. сб. 1945. Т. 16 (125). С. 125-128. [3] Е. В. Шепин // УМН. 1998. Т. 53. № 5. С. 115-212. [4] Е. В. Шепин // УМН. 1976. Т. 31. № 5. С. 191-226. [5] К. Куратовский. Топология. Т. 2. М.: Мир, 1969.

Математический институт

им. В.А. Стеклова РАН
Принято редколлегией 23.09.2003 